Introduction Small Bowel Bacterial Overgrowth (SBBO) has recently been proposed to be prevalent in patients with diarrhoea predominant Irritable Bowel Syndrome (IBS-D). However prevalence figures in studies have varied widely dependant on the diagnostic test used, with low prevalence rates of $4 \%$ using jejunal aspirate and culture, to between $38-84 \%$ using hydrogen breath tests.

This study used the Glucose Hydrogen and Methane Breath Test (GHBT) to determine if there was a relationship between patients with IBS-D symptoms and SBBO. Concurrently any clinical features or baseline laboratory investigations indicative of a high likelihood of SBBO was investigated.

Methods A retrospective analysis of patient hospital records for patients referred to a tertiary GI Physiology Department at Sheffield Teaching Hospitals (STH) between January 1988-2013 for a routine GHBT was conducted. Data was split into two groups. Group 1 included all patients demographics referred for a GHBT (1998-2010) to investigate characteristics predictive of SBBO. Group 2 included patients who fulfilled ROME III criteria for IBS-D who underwent a GHBT (2010-2012). A positive result for SBBO was defined as a rise in hydrogen or methane levels of $\geq 10 \mathrm{ppm}$ over baseline levels.

Results In group 1786 patients were identified (276 male, mean age 54). Overall 175/786 (22.3\%) tests were positive. Laboratory investigations and patient characteristics predictive of a positive result were low vitamin $\mathrm{B}_{12}(\mathrm{p}<0.001)$, low albumin $<30 \mathrm{~g} / \mathrm{dL}$ ( $\mathrm{p}<0.001$ ), concurrent use of a proton pump inhibitor (PPI) ( $\mathrm{p}=0.002)$, previous Bilroth II gastroenterostomy ( $\mathrm{p}$ $<0.001$ ), previous vagotomy ( $\mathrm{p}<0.001$ ), right hemi-colectomy $(\mathrm{p}=0.003)$, coeliac disease $(\mathrm{p}<0.001)$, and small bowel Crohn's disease $(p=0.04)$ and age over 65 years $(p<0.001)$. Symptoms predictive of a positive GHBT included diarrhoea ( $p$ $=0.03)$ and weight loss $(\mathrm{p}<0.01)$.

In group 2 135/834 patients fulfilled ROME III criteria for IBS-D (42 male, mean age 43 years). Overall 26/135 (19.3\%) tests were positive. A significant correlation was found between patients with IBS-D symptoms and SBBO $(\mathrm{p}=0.01)$. Characteristics predictive of SBBO in IBS-D patients were previous small bowel surgery $(p=0.04)$ and blind loop syndrome $(p=0.04)$. Conclusion This study has further highlighted the proposed relationship of IBS-D symptoms and an increased prevalence of SBBO. In IBS-D patients factors predictive of SBBO were previous small bowel surgery and blind loop syndrome. For the sub-cohort of patients attending for a GHBT factors predictive of SBBO were patient age ( $\geq 65$ years), diarrhoea, weight loss, use of a PPI, previous Bilroth II gastroenterostomy, previous vagotomy, right hemi-colectomy, coeliac disease, small bowel Crohn's disease, low Vitamin $\mathrm{B}_{12}$ and low albumin.

Disclosure of Interest None Declared.

\section{PWE-169 PERCUTANEOUS TIBIAL NERVE STIMULATION HAS SUSTAINED BENEFIT AT 1 YEAR IN PATIENTS WITH FAECAL INCONTINENCE}

JB Cowley*, P Waudby, HL O'Grady, G Duthie. Academic Surgical Unit, Hull and East Yorkshire NHS Trust, Hull, UK

\subsection{6/gutjnl-2014-307263.429}

Introduction Faecal incontinence affects up to $18 \%$ of the adult population ${ }^{1}$ with obstetric trauma being the most common risk factor. ${ }^{2,3}$ Multiple techniques are used in its management with neuromodulation becoming increasingly popular. SNS is a safe and effective treatment for FI but has associated morbidity ${ }^{4}$ and costs. ${ }^{5}$ PTNS is an alternative that evolved from the treatment of urinary incontinence, ${ }^{6}$ involving stimulation of the tibial nerve. We aimed to assess functional outcome in patients treated with PTNS initially, post treatment and in those that have completed follow up at 1 year.

Methods All patients undergoing neuromopdulation with PTNS in our institution were included. Treatment regimens of $30 \mathrm{~min}$ PTNS for 12 weeks were undertaken. Patients were assessed with pre, post treatment and in those that qualify at 1 year with validated questionnaires. The questionnaires included the CCFIS and Rockwood FIQOL score. Data was analysed with SPSS using the Wilcoxon signed rank test with significance at $\mathrm{p}<0.05$.

Results 40 patients underwent PTNS, 36 female with median age 60 years (range 34-85). 30 were successful with a trial of PTNS, 9 failing and one lost to follow up. Of successful patients a further 4 were lost to follow up at a year. CCFIS scores were significantly improved in the post treatment group $(\mathrm{n}=38)$ with $\mathrm{z}=-3.75, \mathrm{p}<0.05 \mathrm{r}=-0.43$ and at 1 year $(\mathrm{n}=9) \mathrm{z}=-0.26$, $\mathrm{p}<0.05, \mathrm{r}=-0.63$. Comparison of the individual components of the FIQOL scores demonstrated significant improvement ( $\mathrm{p}$ $<0.05$ ) in lifestyle, coping, depression and embarrassment scores post treatment and at 1 year all other than lifestyle continued to show significance.

Conclusion PTNS shows significant benefit in CCFIS scores and FIQOL scores post treatment and at 1 year. Whilst patient numbers at 1 year are small this treatment is in its infancy and further long term studies will be needed to confirm benefit over time.

\section{REFERENCES}

1 Whitehead WE, Borrud L, Goode PS, et al. Fecal incontinence in US adults: epidemiology and risk factors. Gastroenterology 2009:137:512-517

2 Madoff RD, Williams JG, Caushaj PF. Fecal incontinence. N Engl J Med. 1992;326:1002-1007

3 Zutshi M, Tracey TH, Bast J, Halverson A, Na J. Ten-year outcomeafter anal sphincter repair for fecal incontinence. Dis Colon Rectum 2009:52:1089-1094

4 Dudding TC, Meng Lee E, Faiz O, Pares D, Vaizey CJ, McGuire A, et al. Economic evaluation of sacral nerve stimulation for faecal incontinence. Br J Surg 2008:95: 1155-1163

5 Sprange K, Clift M, Burke M, Whitehead SR, Hutton J. Evidence Review: Sacra Nerve Stimulation for Faecal Incontinence. Healthcare Innovation and Technology Evaluation Centre (HITEC): Derby, 2009

6 McGuire EJ, Zhang SC, Horwinski ER, Lytton B. Treatment of motor and sensory detrusor instability by electrical stimulation. J Urol 1983:129: 78-79

Disclosure of Interest None Declared.

\section{PWE-170 AN AUDIT OF CLINICAL RESPONSE IN PATIENTS WITH IBS TREATED WITH THE LOW FODMAP DIET AT ST MARY'S HOSPITAL}

V Mcgeoch, V Blackwell, L Wigham, JM Hoare*. Gastroenterology, Imperial College London, London, UK

\subsection{6/gutjn--2014-307263.430}

Introduction The low FODMAP diet is a diet used in patients with Irritable Bowel Syndrome. The diet is based on evidence that fermentable oligosaccharides, disaccharides, monosaccharides and polyols (FODMAPs) contribute to symptoms many people experience in Irritable Bowel Syndrome (IBS), including: bloating, flatulence and diarrhoea. ${ }^{1}$ This may be due to their fermentation by bacteria and osmotic effects on the bowel. ${ }^{2}$ The low FODMAP diet aims to reduce these carbohydrates and so reduce symptoms.

Methods The study included all patients treated with the low FODMAP diet at St Marys Hospital between November 2009 
and February 2013. Patients treated with the low FODMAP diet were identified using dietetic records and correspondence. Of the whole study population $(\mathrm{n}=80), 27 \mathrm{did}$ not attend a follow up appointment and 7 were waiting for follow up at the time of the study. This left 46 patients with follow up appointment data. Their records were analysed and symptom data recorded. A comparison between before and after the diet was then made for symptom changes.

Results Following treatment with the low FODMAP diet patients reported a lower incidence of each IBS symptom. When the group was assessed for improvement, the results showed that for each symptom, at least $70 \%$ of patients improved. The biggest improvements were for bloating (93\% improved) and flatulence ( $92 \%$ improved). Average stool consistency reduced from 5.21 to 4.16 on the Bristol Stool Chart. Average stool frequency per day reduced from 3.87 to 1.56 . The diet has been used increasingly each year since 2009 .

Conclusion The majority of patients treated with the low FODMAP diet at St Marys Hospital had symptomatic improvements. In this population it has been effective in treating symptoms that are difficult to treat medically such as; bloating, abdominal pain and flatulence.

\section{REFERENCES}

1 Gibson PR, et al, Food choice as a key management strategy for functional gastrointestinal symptoms. Am J Gastroenterol 2012 May:107(5):657-66

2 Barrett JS, et al, Dietary poorly absorbed, short-chain carbohydrates increase delivery of water and fermentable substrates to the proximal colon, Aliment Pharmacol Ther 2010 Apr;31(8):874-882

Disclosure of Interest None Declared.

\section{PWE-171 ASSESSING THE UTILITY OF KEY MRI PARAMETERS IN CHARACTERISING THE MODE OF ACTION OF A PROVEN EFFECTIVE LAXATIVE, ISPAGHULA}

${ }^{1} \mathrm{~K}$ Murray ${ }^{*},{ }^{2} \mathrm{~L}$ Marciani, ${ }^{2} \mathrm{G}$ Major, ${ }^{1} \mathrm{C}$ Hoad, ${ }^{1} \mathrm{E}$ Cox, ${ }^{3} \mathrm{~A}$ Silos-Santiago, ${ }^{3} \mathrm{C}$ Kurtz, ${ }^{1} \mathrm{M}$ Kaviani, ${ }^{1} \mathrm{JA}$ Paul, ${ }^{1} \mathrm{P}$ Gowland, ${ }^{3} \mathrm{~J}$ Johnston, ${ }^{2} \mathrm{R}$ Spiller. ${ }^{1}$ Sir Peter Mansfield Magnetic Resonance Centre, Nottingham, UK; ${ }^{2}$ NIHR Nottingham Digestive Diseases Biomedical Research Unit, University of Nottingham, Nottingham, UK; ${ }^{3}$ Ironwood Pharmaceuticals Inc., Cambridge MA, USA

10.1136/gutjn-2014-307263.431

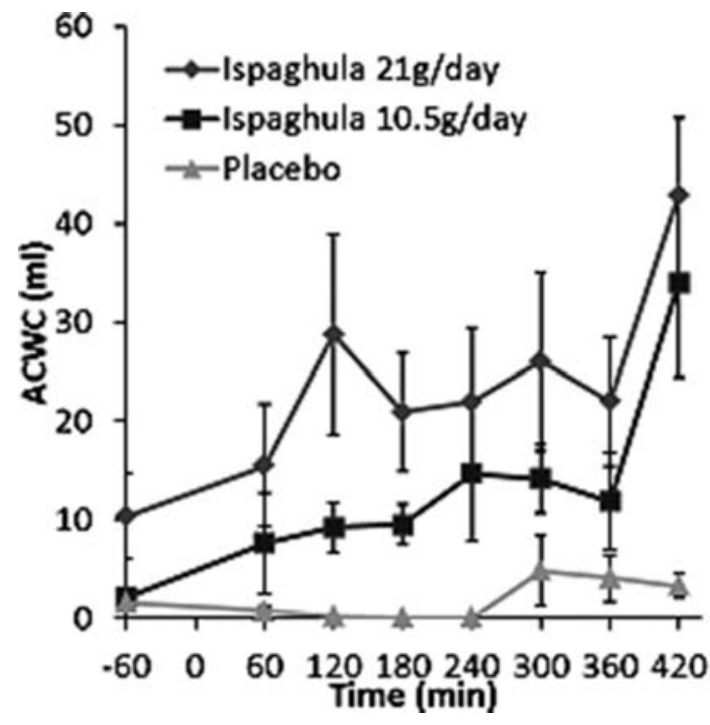

Abstract PWE-171 Figure 1 Time course of freely mobile water in the ascending colon (ACWC) throughout the MRI study day

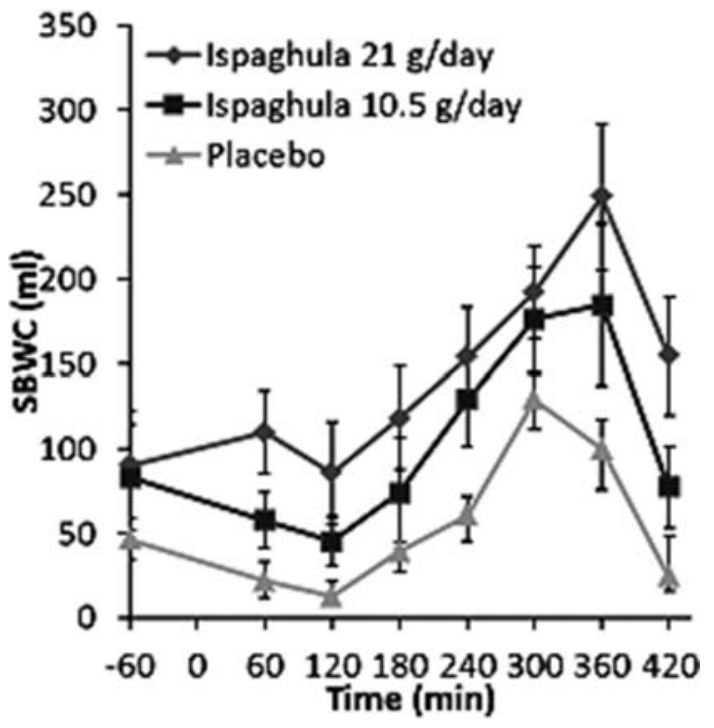

Abstract PWE-171 Figure 2 Time course of the freely mobile water in the small bowel (SBWC) throughout the MRI study day

Introduction Half of the patients who suffer from chronic constipation are dissatisfied with their treatments, which has initiated a recent surge in novel treatments. Assessing the efficacy of these treatments requires techniques that are patient acceptable and non-invasive and allow characterisation of the underlying mode of action. This study assessed the utility of key parameters likely to give insight into laxatives' mode of action including: colonic volumes, transit, ascending colon water content (ACWC) and small bowel water content (SBWC) by means of a randomised double-blind, placebo-controlled, crossover study (RCT) of a known effective laxative, ispaghula.

Methods 16 healthy volunteers $(24 \pm 4$ years old, BMI $23 \pm 4$ $\mathrm{kg} \mathrm{m}^{-2}$ ) participated in this three-way RCT. They took either $21 \mathrm{~g} /$ day of ispaghula, $10.5 \mathrm{~g}$ /day of ispaghula or a placebo daily on days 1-6. On day 5 they swallowed 5 transit marker pills (TMP) filled with a dilute MR contrast agent and on day 6 were scanned serially for $7 \mathrm{~h}$. The TMPs were assigned a weighted average position score (WAPS) based on their location in the bowel. Protocol compliance was assessed and 9 subjects were included in the per protocol analysis.

Results (mean AUC \pm SEM, $n=9$ ) Relative to the placebo, $21 \mathrm{~g} /$ day but not $10.5 \mathrm{~g} /$ day of ispaghula increased the ACWC (Figure 1$)$ significantly $(11 \pm 4$ L.min vs. $1.0 \pm 0.5$ L.min, $\mathrm{p}<$ 0.001), while SBWC (Figure 2) was increased significantly by both doses $(68 \pm 15$ L.min vs placebo $25 \pm 6$ L.min, $p=$ $<0.01)$, and $(49 \pm 11$ L.min, $\mathrm{p}=<0.05)$ respectively. Both doses significantly increased the total colon volume, and there was a significant increase in colon volume at fasted baseline measurement. Ascending colon T1 was also increased by the $21 \mathrm{~g} /$ day dose $(\mathrm{p}<0.01)$, but $24 \mathrm{~h}$ WAPS for transit were not significantly changed by treatment.

Conclusion The volume of water in both the ascending colon and the small bowel are significantly increased by ispaghula and could be useful biomarkers of a laxative effect. Ispaghula also increased colonic volumes and ascending colon T1 without altering transit times, suggesting it or its metabolites does not stimulate motility. These MRI methods could be readily used in assessing the mode of action of a range of novel agents in constipated patients will provide unique information on the mechanisms of action.

Disclosure of Interest None Declared. 